\title{
Valoración de experto sobre la germinación de la semilla de maíz
}

\author{
Expert assessment of corn seed germination \\ Avaliação especializada da germinação de sementes de milho
}

\author{
Ángel Martínez Rengel \\ oswalmar69@gmail.com \\ https://orcid.org/0000-0003-3147-8608 \\ Consejo de la judicatura, Ecuador
}

\author{
Cesar Álvarez Morejon \\ cem.cesaralvarez@gmail.com \\ https://orcid.org/0000-0002-3271-5161 \\ Universidad del Pacifico, Quito-Ecuador
}

\author{
María Torres Cedeño \\ ikatorrescedeno@gmail.com \\ https://orcid.org/0000-0003-1377-6505 \\ Universidad Técnica de Esmeraldas Luis Vargas Torres, \\ Esmeraldas-Ecuador
}

\author{
Nuvia Renteria Valencia \\ nuvia.renteria48@yahoo.com \\ https://orcid.org/0000-0002-1886-1699 \\ Universidad Técnica de Esmeraldas Luis Vargas Torres, \\ Esmeraldas-Ecuador
}

\section{RESUMEN}

El experto independiente, tiene un rol importante como garante de los intereses de usuarios tanto internos como externos. Por ello, el legislador exige su intervención en operaciones en que dichos usuarios pueden ser vulnerables a los intereses de otros. En este artículo se analiza y expone la valoración de experto independiente donde se evidencia, a través de un caso concreto, las anomalías presentadas en la siembra de maíz clon Advanta 9139, compradas a la empresa farmagro; objeto de litigio. Además, se presentan los resultados estadísticos correspondientes. El trabajo realizado tuvo como objeto determinar y evidenciar el porcentaje de germinación de las semillas en un lote de 87 hectáreas, por lo que se aplicó el estadístico de Ji2 con la finalidad de verificar si la semilla germinada presenta relación con las semillas sembradas. Se concluyó que la cantidad de semillas sembradas de maíz clon Advanta 9139 en la hacienda Macavelo, no es igual a la cantidad de semillas germinadas.

Palabras clave: Valoración de experto; Siembra de maíz clon Advanta 9139; Germinación de semillas de maíz; Estadístico de Ji2
ABSTRACT

The independent expert has an important role as guarantor of the interests of both internal and external users. Therefore, the legislator requires their intervention in operations in which said users may be vulnerable to the interests of others. This article analyzes and exposes the assessment of an independent expert where it is evidenced, through a specific case, the anomalies presented in the planting of maize clone Advanta 9139, purchased from the Farmagro company; subject of litigation. In addition, the corresponding statistical results are presented. The purpose of the work carried out was to determine and demonstrate the percentage of germination of the seeds in a lot of 87 hectares, for which the Ji2 statistic was applied in order to verify if the germinated seed presents a relationship with the sown seeds. It was concluded that the quantity of seeds sown of maize clone Advanta 9139 in the Macavelo farm is not equal to the quantity of germinated seeds.

Key words: Expert assessment; Advanta 9139 clone maize sowing; Maize seed germination, Ji2 statistic
RESUMO

O perito independente tem um papel importante como fiador dos interesses dos usuários internos e externos. Portanto, o legislador exige sua intervenção em operações em que os referidos usuários possam ser vulneráveis aos interesses de terceiros. Este artigo analisa e expõe a avaliação de um perito independente onde são evidenciadas, através de um caso específico, as anomalias apresentadas no plantio do milho clone Advanta 9139, adquirido da empresa Farmagro; objeto de litígio. Além disso, são apresentados os resultados estatísticos correspondentes. $\mathrm{O}$ objetivo do trabalho realizado foi determinar e demonstrar a porcentagem de germinação das sementes em um lote de 87 hectares, para o qual foi aplicada a estatística Ji2, a fim de verificar se a semente germinada apresenta relação com as sementes semeadas. Concluiu-se que a quantidade de sementes semeadas do milho clone Advanta 9139 na Fazenda Macavelo não é igual à quantidade de sementes germinadas.

Palavras-chave: Avaliação de especialistas; Semeadura de clones de milho Advanta 9139; Germinação de sementes de milho; Estatística Ji2 


\section{INTRODUCCIÓN}

El experto independiente tiene un papel clave como garante de los intereses de usuarios tanto internos como externos. Por ello, el legislador exige su intervención en operaciones en que dichos usuarios pueden ser vulnerables a los intereses de otros. Su función principal, por tanto, es la de elaborar un informe que aporte objetividad a la operación, y un conocimiento más crítico y técnico. También, de acuerdo con el legislador, da discrecionalidad al experto a la hora de elegir el mejor método de valoración en función de las circunstancias concretas del caso. No obstante, dado que no es arbitraria esta decisión, se deben estudiar los distintos métodos que ofrece la doctrina, valorando sus ventajas e inconvenientes y su pertinencia en cada caso concreto (1).

Por ello, en este artículo, a través de un caso práctico, se analiza y expone un trabajo donde se evidenciar las anomalías presentadas en la siembra de maíz clon Advanta 9139 compradas a la empresa farmagro, objeto de litigio, y se presentan los resultados estadísticos correspondientes.

Elmaízesunodelostresprincipalescereales producidos en el mundo junto con el trigo y el arroz, además es un cultivo cosmopolita, lo cual le ha permitido desarrollarse en una infinidad de condiciones climáticas, edáficas, sociales y ecológicas. Muchos son los factores que influyen sobre la productividad del maíz, iniciando propiamente desde la planeación del cultivo hasta su cosecha. En este sentido, al contar con una rápida y uniforme germinación, se establece el primer escenario para lograr el rendimiento potencial al final del ciclo de producción (2).

La agricultura moderna demanda semilla de alta calidad, siendo esta el principal insumo que en la agricultura debe cumplir con diferentes atributos, entre estos se encuentran: la calidad genética, fisiológica, física y sanitaria. Las pruebas de germinación y de viabilidad han sido utilizadas ampliamente en la evaluación de la calidad de las semillas, cabe destacar que la calidad fisiológica hace referencia a mecanismos intrínsecos de la semilla que determinan su capacidad de germinación, la emergencia y el desarrollo de aquellas estructuras esenciales para producir una plántula normal bajo condiciones favorables. Sin embargo, en los últimos años se ha dado énfasis en la medición de otros parámetros, tales como: el vigor y las variables asociadas con este parámetro (3).

Por tal motivo, es de fundamental importancia realizar un control de calidad, tal como lo señala Velázquez,(4), a fin de minimizar los riesgos que implica utilizar semillas que no tienen una adecuada capacidad para producir buenas cosechas, y dentro de este proceso se ven involucrados los diferentes métodos útiles y confiables para determinar las principales características de una semilla de alta calidad, es decir; cuando es pura, tiene germinación, alto vigor, está libre de enfermedades y tiene buena confirmación.

Esta práctica es utilizada en forma cada vez más frecuente, ya que en general y por diversas razones, en todos los cultivos en que se requieren semillas se producen problemas 
que afectan tanto a productos de semillas como a los agricultores. Este aspecto adquiere mayor relevancia, al considerar que la comercialización de semilla en el país y en el exterior es cada vez más exigente en la calidad demandada. Es importante destacar que un oportuno control de calidad de la semilla repercutirá directamente en la producción y es de conocimiento que el valor de un análisis de semilla tiene una incidencia bajísima en los costos directos comparando con los futuros resultados que se obtendrán (5).

Así pues, que la calidad fisiológica de la semilla abarca la suma de todas las propiedades o características, las cuales determinan el nivel potencial del comportamiento de las semillas y el establecimiento del cultivo. Los componentes de la calidad de la semilla incluyen los aspectos genéticos, físicos, fisiológicos y sanitarios (microorganismos e insectos) (4).

La prueba de germinación estándar es el procedimiento más común para evaluar la calidad fisiológica de un lote de semillas. No obstante, debido a que esta prueba se realiza bajo condiciones óptimas para cada especie, en la práctica se ha demostrado sobreestimar el comportamiento de las semillas y, además, resulta deficiente para discriminar lotes de semillas en relación con la rapidez y uniformidad de germinación (6).

Tal como indican (3) es necesario evaluar el vigor, la definición de vigor es relativamente novedosa en comparación con la germinación. El vigor de la semilla es un parámetro muy importante puesto que permite identificar las diferencias entre la germinación y la emergencia en campo, principalmente cuando las condiciones del campo pueden ocasionar estrés. Dentro de la utilidad práctica de los ensayos de vigor de semillas, se encuentra su uso en los programas de mejoramiento genético para el desarrollo de cultivos con mejor comportamiento de las semillas. Tiene además aplicaciones en el estudio de los aspectos de la producción de semilla, cosecha, acondicionamiento y procedimientos de almacenamiento. Actualmente los ensayos de germinación y de vigor, se están usando para determinar el efecto que ejercen los tratamientos con nano partículas (NPs), nanotubos de carbono (NTC), grafito u óxido de grafema, aplicados a semillas, en el proceso de germinación y vigor de semillas y plántulas.

En opinión de Burbano (7) es clave destacar que un buen manejo del control de la calidad de la semilla influye directamente sobre su valor comercial. La prueba de germinación estándar es el procedimiento más común para evaluar la calidad fisiológica de un lote de semillas y sirve para determinar en gran medida la viabilidad de la semilla, que tiene la capacidad de producir una plántula normal, la cual establece la capacidad de germinación.

Para esteautor, lassemillas debenserdealta calidad, limpias y compradas de una compañía de semillas acreditada. Con pocas excepciones, es un requisito que los productores orgánicos certificados usen únicamente semillas y plántulas certificadas como orgánicas. Todas las semillas bien desarrolladas contienen un embrión y en la mayoría de las especies de 
plantas una reserva de alimentos envuelta en una cutícula que cubre la semilla. Las semillas generalmente "se despiertan" y germinan bien cuando el suelo esta húmedo y las condiciones de temperatura son favorables para que éstas puedan crecer. Pero no todas las semillas tienen los mismos requisitos de germinación, por lo que es importante conocer lo que cada tipo de semilla necesita.

Todas las semillas necesitan una humedad adecuada y oxígeno para germinar. Es importante que haya un buen contacto entre la semilla y el suelo. Para plantar directamente en el suelo se recomienda un almácigo con un suelo de textura fina que no se compacte mucho. Para los almácigos de invernadero el medio de cultivo debe ser sin tierra, pero húmedo y esponjoso. El suelo o el medio de cultivo deben tener suficiente humedad para que las semillas puedan absorber el agua para comenzar el proceso de germinación. Como cualquier otro organismo vivo las semillas también necesitan oxígeno para respirar, y el oxígeno viene del aire que circula en el suelo cuando no es compacto o demasiado húmedo.

Sin embrago, no todas las semillas tienen los mismos requisitos de luz. La mayoría de las semillas germinan mejor bajo condiciones de oscuridad y la luz puede impedir su germinación. Sin embargo, algunas otras necesitan luz para germinar. Pero una vez que las semillas han germinado y han brotado del suelo o del medio de cultivo como plántulas, todas necesitan de la luz del sol para crecer. Sabemosquelassemillas necesitancondiciones apropiadas para germinar rápidamente. Ya sea que las semillas se planten en bandejas en los invernaderos o directamente en el campo, la meta es que todas las semillas germinen al mismo tiempo o cerca del mismo tiempo y que crezcan a la misma velocidad. Una bandeja de trasplantes uniformes es más fácil de manejar y le llevará a obtener una mejor cosecha. Una germinación dispareja debido a un crecimiento lento, a diferencias de humedad en el suelo o en las temperaturas, o a diferencias en la profundidad al plantar las semillas puede resultar en plántulas de diferentes tamaños, la mitad estará lista para trasplantarse directamente al suelo y la otra mitad estará demasiado chica, con las raíces enrolladas sin que las raíces salgan fácilmente de sus celdas (8).

No se puede controlar las condiciones del campo como se puede hacer dentro de los invernaderos, pero aun así se pueden tomar medidas para asegurar que las semillas plantadas directamente en el campo germinen uniformemente. Una cama de suelo de textura fina le provee buenas condiciones de crecimiento a las semillas, un buen contacto entre suelo y semilla y la habilidad de plantarlas a una profundidad uniforme. Si se plantan cuando las temperaturas del suelo están casi óptimas acelerará la germinación y la emergencia de las plántulas. A veces con la prisa de sembrar en la primavera, las semillas se siembran en suelos que aún están muy fríos. Esto puede resultar en una germinación lenta, plántulas débiles y enfermizas y su muerte. Es mejor retrasar la siembra hasta que los suelos se hayan calentado (9). 
Sin embargo, los componentes de calidad pueden ser afectados adversariamente durante la producción, beneficio, almacenamiento y transporte de las semillas (9). De tal manera, es fundamental realizar un control de calidad y dentro de este se ven involucrados los diferentes métodos útiles y confiables para determinar las principales características de lotes de semillas con buena calidad, con la finalidad de cumplir con los estándares de germinación y vigor. Este aspecto adquiere mayor relevancia aún, ya que el mercado de las semillas ha experimentado importantes cambios como la globalización y el incremento de su valor (10).

\section{MATERIALES Y MÉTODOS}

Según lo que señala Surco Seguros, (5) el Perito procede a realizar el peritaje correspondiente únicamente si ha recibido aviso de siniestro por parte del afectado. Una vez que el Perito recibe la solicitud de peritar el siniestro el profesional se pondrá en contacto con el afectado para avisarle al mismo de que ya ha tomado contacto con su denuncia, y coordinar la posible fecha de inspección, siendo atribución exclusiva del Perito decidir la fecha de inspección, la que responderá a la posibilidad de efectuar una correcta tarea técnica.

El Perito deberá comprobar que la información recibida en la denuncia de siniestro (ubicación, superficie, cultivo, etc.) coincida con el cultivo que inspeccionará. En caso contrario, deberá dejar constancia en el informe; e incluso puede negarse a realizar el peritaje. Para la evaluación de daños es obligatoria la confección de planillas de campo con el registro de los datos obtenidos, así como la confección del plano de la superficie asegurada incluyendo los puntos de muestreo y todo dato que el Perito estime pertinente. El procedimiento seguido se puede resumir en términos de:

- Constatar la ubicación, cultivo y superficie asegurada. Esta verificación se efectuará a partir de la documentación suministrada.

- Dibujar -si es necesario- el plano sobre el formulario de informe, ubicando de ser necesario los puntos de muestreo (numerados), con indicación de distancias. Más adelante se incluyen instrucciones acerca del procedimiento de muestreo.

- En cada sitio muestreado completar la planilla correspondiente, de acuerdo a la metodología indicada.

- Determinar superficies afectadas por daño dentro de la chacra, para -si es necesario construir un mapa de daños que permita delimitar los distintos sectores. Cada sector abarcará los sitios de muestreo cuyos datos de daño no difieren significativamente entre sí; el daño correspondiente al sector individualizado se obtendrá promediando las observaciones realizadas dentro del mismo.

- Cálculo de las superficies afectadas. Se utilizará GPS o métodos convencionales de cálculo de superficies, dejando constancia de la escala utilizada y el procedimiento utilizado. 
En este artículo, se analiza y expone la valoración de un experto independiente, donde en atención a todo lo anterior, se realizó este procedimiento.

A los treinta días del mes de diciembre del 2019 a las 10 h00 AM, en la Hacienda MACAVELO ubicada en la Parroquia Patricia Pilar margen derecho km47 vía a Quevedo, del cantón Buena Fe, provincia de los Ríos. En función del Código Orgánico de Procesos; "COGEP”; PAZ\&LARREA LARREAPAZ CIA.LTDA, solicita como perito al señor Ing. Ángel Oswaldo Martínez Rengel, titular con \# de calificación 2546383 del Consejo de la Judicatura; la pericia en la Hacienda MACAVELO, quien es posesionado de su cargo y promete desempeñarlo en legal y debida forma con el objeto de:

- Realizar un recorrido para evidenciar las anomalías presentadas en la siembra de maíz clon ADVANTA 9139 compradas a la empresa FARMAGRO, objeto de la Litis.

- Determinar y evidenciar el porcentaje de germinación de las semillas de maíz clon ADVANTA9139 en un lote de 87 hectáreas.

- Identificar variables que inciden en un adecuado porcentaje de germinación de la semilla de maíz.

- Realizar un recorrido para determinar la posesión del predio, indicando cultivos o mejoras existentes como viviendas, objeto de la Litis.

\section{RESULTADOS Y DISCUSIÓN}

El presente informe pericial se basa, en la revisión in situ de parcelas con problemas de germinación y análisis de documentación existente sobre la siembra de semillas de maíz clon "ADVANTA 9139"compradas a la empresa FARMAGRO, objeto de la Litis. Para la revisión de las parcelas se conformó un equipo de profesionales, dos por parte de la empresa afectada por la presunta mala germinación de la semilla y tres por parte de la empresa FARMAGRO, vendedora de la semilla.

Además, para constancia de las observaciones hechas se procedió a realizar un Acta de Acuerdos en la que firmaron las partes involucradas como parte del compromiso ahí resuelto, Además, se observó variables biométricas y agrícolas para evidenciar su comportamiento se hizo el análisis de algunas de las variables biométricas y agrícolas. (Tablas $1,2,3,4,5)$. 
Tabla 1. Semillas germinadas, sembradas, y plantas afectadas posiblemente por herbicida, en la hacienda Macavelo.

\begin{tabular}{lcccc}
\hline DESCRIPCION & LINEAS & $\begin{array}{c}\text { TOTAL } \\
\text { SEMILLAS } \\
\text { GERMINDAS }\end{array}$ & $\begin{array}{c}\text { PLANTAS } \\
\text { AFECTADAS }\end{array}$ & $\begin{array}{c}\text { SEMILLAS } \\
\text { SEMBRADAS }\end{array}$ \\
\hline & 1 & 37 & & 110 \\
& 2 & 23 & 14 & 110 \\
MUESTRA 1 área 192m2 & 3 & 99 & 29 & 110 \\
(20m de largo por 12 & 4 & 82 & 41 & 110 \\
de 0,8m entre fila) & 5 & 61 & 26 & 110 \\
& 6 & 61 & 31 & 110 \\
& 7 & 50 & 27 & 110 \\
& 8 & 23 & 15 & 110 \\
& 9 & 36 & 18 & 110 \\
& 10 & 109 & 9 & 110 \\
TOTAL & 11 & 110 & 14 & 110 \\
\hline
\end{tabular}

Tabla 2. Totales de semillas germinadas, sembradas, y plantas afectadas posiblemente por herbicida, en la hacienda Macavelo.

\begin{tabular}{lcccc}
\hline \multicolumn{1}{c}{ DESCRIPCION } & LINEAS & $\begin{array}{c}\text { TOTAL } \\
\text { SEMILLAS } \\
\text { GERMINDAS }\end{array}$ & $\begin{array}{c}\text { PLANTAS } \\
\text { AFECTADAS }\end{array}$ & $\begin{array}{c}\text { SEMILLAS } \\
\text { SEMBRADAS }\end{array}$ \\
\hline & 1 & 66 & 10 & 110 \\
MUESTRA 2 área 96m2, & 2 & 11 & 7 & 110 \\
(20m de largo por & 3 & 101 & 10 & 110 \\
6 de 0,8m entre fila) & 4 & 48 & 10 & 110 \\
& 5 & 66 & 23 & 110 \\
& 6 & 65 & 17 & 110 \\
\hline TOTAL & & $\mathbf{3 5 7}$ & $\mathbf{7 7}$ & $\mathbf{6 6 0}$ \\
\hline
\end{tabular}


Tabla 3. Indica muestra 1 , con totales de semillas germinadas, sembradas, y plantas afectadas posiblemente por herbicida, en la hacienda Macavelo.

\begin{tabular}{lcccc}
\hline \multicolumn{1}{c}{ DESCRIPCION } & LINEAS & $\begin{array}{c}\text { TOTAL } \\
\text { SEMILLAS } \\
\text { GERMINDAS }\end{array}$ & $\begin{array}{c}\text { PLANTAS } \\
\text { AFECTADAS }\end{array}$ & $\begin{array}{c}\text { SEMILLAS } \\
\text { SEMBRADAS }\end{array}$ \\
\hline & 1 & 90 & 6 & 110 \\
MUESTRA 3 área 96m 2, & 2 & 107 & 3 & 110 \\
(20m de largo por & 3 & 18 & 7 & 110 \\
6 de 0,8m entre fila) & 4 & 65 & 6 & 110 \\
& 5 & 77 & 3 & 110 \\
\hline TOTAL & 6 & 12 & 7 & 110 \\
\hline
\end{tabular}

Tabla 4. Indica muestra 1 , con totales de semillas germinadas, sembradas, y plantas afectadas posiblemente por herbicida, en la hacienda Macavelo.

\begin{tabular}{lcccc}
\hline \multicolumn{1}{c}{ DESCRIPCION } & LINEAS & $\begin{array}{c}\text { TOTAL } \\
\text { SEMILLAS } \\
\text { GERMINDAS }\end{array}$ & $\begin{array}{c}\text { PLANTAS } \\
\text { AFECTADAS }\end{array}$ & $\begin{array}{c}\text { SEMILLAS } \\
\text { SEMBRADAS }\end{array}$ \\
\hline & 1 & 71 & 6 & 110 \\
MUESTRA 4 área 96m2, & 2 & 37 & 3 & 110 \\
(20m de largo por & 3 & 101 & 7 & 110 \\
6 de 0,8m entre fila) & 4 & 91 & 6 & 110 \\
& 5 & 106 & 3 & 110 \\
\hline TOTAL & 6 & 83 & 7 & 110 \\
\hline
\end{tabular}

Tabla 5. Variables biométricas y agrícolas analizadas en la hacienda Macavelo.

\begin{tabular}{llcc}
\hline \multicolumn{1}{c}{ DESCRIPCION } & \multicolumn{1}{c}{ MEDIDA } & CUMPLE & NO CUMPLE \\
\hline TIPO DE SEMILLA & SEMILLA CERTIFICADA & $\mathrm{X}$ & \\
PREPARACION DE TERRENO & LABORES CULTURALES & $\mathrm{X}$ & \\
FORMA DE SIEMBRA & MECANICA & & \\
PROFUNDIDAD DE SIEMBRA & 2PLG & $\mathrm{X}$ \\
USO DE FICHA TECNICA DE CULTIVO & GUIA DE PROVEEDOR DE SEMILLA & $\mathrm{X}$ \\
CANTIDAD DE SEMILLAS SEMBRADAS & 68750 PLANTAS/HA & $\mathrm{X}$ & \\
CANTIDAD DE SEMILLAS GERMINADAS & 38000 PLANTAS/HA & & $\mathrm{X}$ \\
\hline
\end{tabular}


Como se observa en la Tabla 5 la cantidad de semillas sembradas presenta diferencias con la cantidad de semillas germinadas, por lo que esta variable NO CUMPLE, con el valor correspondiente. Por lo que se aplicó el estadístico de Ji2 con la finalidad de verificar si la variable semilla germinada presenta relación con variable semilla sembrada. Para el cálculo del estadístico Ji2 se empleó los siguientes valores ubicados en la Tabla 6.

Tabla 6. Prueba JI2.

\begin{tabular}{lcccc}
\hline & LINEAS & $\begin{array}{c}\text { SEMILLAS } \\
\text { GERMINDAS }\end{array}$ & $\begin{array}{c}\text { PLANTAS } \\
\text { AFECTADAS }\end{array}$ & $\begin{array}{c}\text { SEMILLAS } \\
\text { SEMBRADAS }\end{array}$ \\
\hline \multirow{3}{*}{ PARCELAS } & P1 & 759 & 1320 & 2079 \\
& P2 & 357 & 660 & 1017 \\
& P3 & 369 & 660 & 1029 \\
TOTAL & P4 & 489 & 660 & 1149 \\
\hline
\end{tabular}

Las Hipótesis empleadas fueron:

Hipótesis Nula; Ho: La cantidad de semillas sembradas de maíz clon "ADVANTA 9139 en la Hacienda MACAVELO, es igual a la cantidad de semillas germinadas.

Hipótesis alternativa; Ha: La cantidad de semillas sembradas de maíz clon "ADVANTA 9139 en la Hacienda MACAVELO, NO es igual a la cantidad de semillas germinadas.

Luego de aplicar el estadístico de Ji2 con un $95 \%$ de confianza se concluye que se acepte la $\mathrm{Ha}$, que dice La cantidad de semillas sembradas de maíz clon "ADVANTA 9139 en la Hacienda MACAVELO, NO es igual a la cantidad de semillas germinadas. Y se rechaza el $\mathrm{Ho}$, que dice La cantidad de semillas sembradas de maíz clon "ADVANTA 9139 en la Hacienda MACAVELO, es igual a la cantidad de semillas germinadas
La semilla de maíz clon "ADVANTA 9139 fue comprada legalmente a la empresa FARMAGRO.

La empresa Paz\&Larrea Larrepaz Cia.Ltda, cumplió con el estándar de ficha técnica de manejo agrícola del cultivo según variables biométricas y agrícolas revisadas.

Luego de aplicar el estadístico de Ji2 con un $95 \%$ de confianza se concluye que se acepte la $\mathrm{Ha}$, que dice La cantidad de semillas sembradas de maíz clon “ADVANTA 9139 en la Hacienda MACAVELO, NO es igual a la cantidad de semillas germinadas. $Y$ se rechaza el Ho, que dice La cantidad de semillas sembradas de maíz clon "ADVANTA 9139 en la Hacienda MACAVELO, es igual a la cantidad de semillas germinadas. 
Debe cumplirse con todo lo acordado en Acta de Acuerdos en la que firmaron las partes involucradas como parte del compromiso ahí resuelto.

\section{CONCLUSIONES}

La oportunidad de compartir la experiencia vivida en el trabajo de campo reportado en este artículo, ha permitido a quien escribe hacer un aporte en cuanto a la valoración de experto sobre la germinación de la semilla de maíz, no sólo para cuantificar estadísticamente un hecho, más que ello para estimar la utilidad de las valoraciones hechas por el experto independiente.

En primer lugar, plantea que la intervención del experto independiente fe necesaria en aquellos casos analizados y tasados por Ley y estos casos tienen como característica común que son situaciones en las que un socio o un tercero pueden estar en una situación de vulnerabilidad, en la que sus intereses pueden verse perjudicados.

En segundo lugar, el informe del experto independiente al final aportó objetividad, independencia y conocimientos técnicos específicos, que se traduce en información muy útil para los agentes afectados. Debido a que, su función principal es como protector de los intereses de usuarios tanto internos como externos.

Por último, lo que el experto independiente pudo valorar estadísticamente, con las pruebas utilizadas en la evaluación de la calidad de las semillas, puede minimizar los riesgos que implica utilizar semillas que no tienen una adecuada capacidad para producir buenas cosechas. Por ello, es fundamental realizar un control de calidad donde estén involucrados los diferentes métodos útiles y confiables para determinar las principales características de una semilla de alta calidad. No estando exenta la semilla de maíz, por lo que se constata que la valoración del experto es de vital importancia. Porque como se indicó anteriormente, la agricultura moderna demanda semilla de alta calidad, siendo el maíz es uno de los tres principales cereales producidos en el mundo junto con el trigo y el arroz, además es un cultivo cosmopolita, lo cual le ha permitido desarrollarse en una infinidad de condiciones climáticas, edáficas, sociales y ecológicas.

\section{REFERENCIAS BIBLIOGRÁFICAS}

1.Serrano M.El papel del experto independiente en la valoración de empresas. Comparativa de métodos de valoración. 2016. Recuperado de https://zaguan.unizar.es/record/56456/files/ TAZ-TFG-2016-2644.pdf

2. Ramos F. Maíz, trigo y arroz. Los cereales que alimentan al mundo (2013). Recuperado de http://eprints.uanl.mx/3649/1/maiztrigoarroz. pdf

3. García-López J, Ruiz-Torres N, LiraSaldivar R, Vera-Reyes I, y Méndez-Argüello B. Técnicas Para Evaluar Germinación, Vigor y Calidad Fisiológica de Semillas Sometidas a Dosis de Nanopartículas. 2016 Recuperado de https://ciqa.repositorioinstitucional. $\mathrm{mx} / \mathrm{jspui} / \mathrm{bitstream/1025/334/1/}$ T\%C3\%A9cnicas\%20Para\%20Evaluar\%20 Germinaci\%C3\%B3n\%2C\%20Vigor\%20y\%20 Calidad $\% 20$ Fisiol\%C3\%B3gica\%20de\%20 Semillas\%20Sometidas\%20a\%20Dosis\%20 de\%20Nanopart\%C3\%ADculas.pdf 
4. Velázquez $\mathrm{H}$. Estudio fisiológico en familias prolíficas de un lote de producción de semilla de la variedad de maíz JAGUAN. Tesis de Maestría Profesional, especialidad en Granos y Semillas. Universidad Autónoma Agraria Antonio Narro. Saltillo, Coahuila. 2014

5. Surco Seguros. Procedimientos de Evaluación de Daños. 2018. Recuperado de https://www. surco.com.uy/images/pdf/Agro/MANUALPERITAJE-2018-compress.pdf

6. McDonald M. Assessment of seed quality. HortScience, Alexandria, v. 1916; (15)6:784-788

7. Burbano E, Papel de los diferentes controles en la producción de semilla de calidad: certificación, bioseguridad, derechos de obtentor. Taller Principios Organizacionales, Tecnológicos y Biológicos en el Manejo Moderno de Semillas de Alta Calidad. Centro Internacional de Agricultura Tropical, CIAT. Colombia. DANE (Departamento Administrativo 2013
8. Doria J. generalidades sobre las semillas: su producción, conservación y almacenamiento. 2010 Recuperado de http://scielo.sld.cu/scielo.php?script=sci_ arttext\&pid=S0258-59362010000100011

9. Méndez J. Ysavit L, y Merazo J. Uso de agua caliente para evaluar la calidad de la semilla de maíz (Zea mays L.) Departamento de Agronomía. Escuela de Ingeniería Agronómica. Núcleo de Monagas. Universidad de Oriente, Campus los Guaritos. Maturín, Estado Monagas, Venezuela. 2007; 8 p

10. Contreras S. The international seed industry. In: Proceedings International Seed Seminar: Trade, Production and Technology. Edts. M. 2012:1-9 